P Gli articoli di questa sezione sono sottoposti a referaggio doppiamente cieco (double blind peer review process) e seguono gli standard in uso per le pubblicazioni scientifiche a livello internazionale ed accettati dalle principali banche dati citazionali

\title{
Orientare l'organizzazione dei Servizi verso un modello efficace e sicuro di trattamento del dolore con farmaci oppiacei: I'esperienza della Casa di Reclusione di Padova
}

\author{
Debora Lageder*, Gaetano Morgillo*, Livia Zuliani*, Giordano B. Padovan*, \\ Jessica Lorenzon*, Felice A. Nava*
}

\begin{abstract}
SUMMARY
- In prison pain assumes a disproportionate magnitude and it is not generally adequately treated. The reasons are often due to the fear to prescribe to detainees opioid analgesic agents for the risk of misuse and diversion. The purpose of this observational study, carried out on 598 detainees in the sentence prison of Padua, was to identify the obstacles and barriers to the use opioid analgesic drugs for pain management and to find recommendations for their implementation and rational use.

The study showed an inappropriate prescription of analgesic drugs and misuse and diversion phenomena, especially in detained drug users.

Recommendations, procedures and guidelines for the pain treatment should be adopted in all prisons.
\end{abstract}

Keywords: Prison, Pain, Opioids, Misuse, Diversion, Addiction.

Parole chiave: Carcere, Dolore, Oppioidi, Misuso, Diversione, Dipendenza.

\section{Introduzione}

Il dolore è un sintomo comune causato da diverse condizioni patologiche. Differenti possono essere i meccanismi generatori del dolore, che può essere influenzato da diversi fattori che possono essere di natura ambientale, familiare, sociale e culturale e possono condizionare anche l'esperienza soggettiva della sensazione dolorosa.

Per l'Organizzazione Mondiale della Sanità (OMS) 1 persona su 5 soffre di dolore cronico con ricadute devastanti sulla sfera fisica, emotiva e sociale dell'individuo.

Una recente indagine condotta in Europa su oltre 45 mila soggetti ha evidenziato che il mancato trattamento del dolore cronico determina enormi costi sociali; basti pensare che il $61 \%$ del campione esaminato lamentava una riduzione della capacità lavorativa e il $19 \%$ era stato costretto a lasciare il lavoro a causa della persistenza del dolore (Breivik, Collett, Ventafridda, Cohen, \& Gallacher, 2006). La metà dei soggetti facenti parte di questo studio soffriva di dolore da un periodo superiore ai 7 anni e nel $46 \%$ dei casi da oltre 10 anni (Breivik et al., 2006).

* Unità Operativa Sanità Penitenziaria, ULSS 6 Euganea, Padova.
Nonostante gli sforzi effettuati negli ultimi decenni per implementare i trattamenti, il dolore, specie quello cronico non neoplastico, continua a essere un'epidemia nascosta (Griffin et al., 2016).

Gestire con successo un caso di dolore cronico utilizzando i farmaci oppiacei significa realizzare un'analgesia adeguata riducendo effetti collaterali e indesiderati (Davis \& Carr, 2016).

\section{Punti critici nella gestione del dolore negli Istituti Penitenziari}

Gli studi dimostrano che all'interno degli Istituti Penitenziari la tipologia di dolore più diffuso è il dolore cronico di natura non neoplastica (Chronic Non-Cancer Pain - CNCP) (Chou et al., 2009; Croft \& Mayhew, 2015). Esso è caratterizzato da una durata del dolore maggiore di tre mesi dovuto a varie cause fra cui patologie muscoloscheletriche, fibromialgia, osteoartrite e artrite reumatoide. Nella definizione di CNCP si escludono condizioni come la cefalea, l'emicrania, I'angina pectoris, il dolore neoplastico e quello dovuto a patologie specifiche come la sclerosi multipla (Reid et al., 2011). Ad ogni modo il CNCP è in continuo incremento nella popolazione detenuta; 
del resto la letteratura indica che il CNCP è spesso associato ad una co-morbidità sia di tipo psichiatrica che fisica (Fazel, Hope, O'Donnell, \& Jacoby, 2001; Singleton, Bumpstead, O’Brien, Lee, \& Meltzer, 1998) ed allo sviluppo in alcuni casi di craving nei confronti della terapia (Martel et al., 2016; Wasan et al., 2009).

Non esistono dati epidemiologici certi sulla prevalenza del dolore negli Istituti Penitenziari. Un recente studio inglese ha messo in evidenza che il $20 \%$ dei detenuti riceve un trattamento per il dolore per una durata maggiore di 3 mesi e di questi il 44\% riceve una terapia oppiacea anche di lunga durata (Croft \& Mayhew, 2015). I limiti maggiori per la diffusione della terapia oppiacea all'interno delle carceri sono i fenomeni di misuso e diversione (Levy, 2012; Wasan et al., 2009), specie fra la popolazione psichiatrica e consumatrice di sostanze (Singleton et al., 1998; Turk, Swanson, \& Gatchel, n.d.).

Fra gli obiettivi primari per il trattamento del dolore in carcere vi è il miglioramento della fase di valutazione e di assessment, dell'appropriatezza prescrittiva e della sicurezza terapeutica. In questo contesto appare anche particolarmente rilevante sviluppare una forte "alleanza terapeutica" con il paziente, in modo da gestire le componenti emotive e percettive del dolore che sono spesso elevate nei contesti detentivi (NHS, 2015a).

Una difficoltà aggiuntiva in ambito penitenziario è la necessità di evitare I'utilizzo di farmaci che possono più frequentemente sviluppare fenomeni di misuso e diversione (Department of Health, 2007). Per questa ragione i contesti carcerari necessitano dello sviluppo di percorsi diagnostici terapeutici o di linee guida per il trattamento del dolore, specie per le popolazioni speciali come i consumatori di sostanze, i pazienti psichiatrici, gli immigrati e le donne.

L'esperienza inglese suggerisce che il 75\% delle carceri ha linee guida per il dolore ma solo 1 su 5 di queste linee guida vengono applicate nella pratica clinica per una difficoltà legata al contesto (fra cui ad es. il trasferimento frequente ed improvviso dei detenuti da un istituto penitenziario ad un altro) (Public Health England, 2013). Un altro limite importante presente nei contesti penitenziari è la difficoltà di sviluppare programmi di trattamento integrati con interventi di tipo comportamentale, educativo e psicologico.

Riassumendo, possiamo affermare che il mancato o inadeguato trattamento del dolore nella popolazione detenuta rappresenta un problema di sanità pubblica di consistente dimensione e che può determinare conseguenze negative sia sulla sfera sociale che su quella sanitaria.

\section{L'esperienza sull'uso dei farmaci oppiacei per il trattamento del dolore nella Casa di Reclusione di Padova}

Presso la Casa di Reclusione di Padova si sono valutati i dati epidemiologici e le modalità prescrittive dei farmaci analgesici oppiacei nella popolazione detenuta al fine di individuare elementi di criticità e di miglioramento per la pratica clinica, volto ad implementare l'accesso al trattamento e I'appropriatezza e la sicurezza prescrittiva.

Lo studio è stato condotto sui pazienti che sono stati trattati per il dolore con farmaci oppiacei attraverso I'analisi delle cartelle, della documentazione clinica e della scheda unica di terapia.

Lo studio è stato condotto sull'intera popolazione detenuta $(\mathrm{n}=$ 598) presente presso la Casa di Reclusione al 30/06/16.

I farmaci oppiacei disponibili per la farmacia della Casa di Reclusione per il trattamento del dolore sono rappresentati in Tabella 1:

\section{Tab. 1 - Farmaci oppiacei disponibili per la terapia del dolore}

\begin{tabular}{ll}
\hline Oppiodi deboli & $\begin{array}{l}\text { Paracetamolo + Codeina } \\
\text { Tramadolo }\end{array}$ \\
\hline Oppiodi forti & Metadone \\
& Buprenorfina \\
Buprenorfina + Naloxone & Ossicodone \\
Ossicodone + Paracetamolo & Ossicodone + Naloxone \\
& Fentanyl \\
Morfina \\
Tapentadolo
\end{tabular}

Le caratteristiche della popolazione in studio sono rappresentate in Tabella 2.

L'analisi comparativa tra il gruppo di detenuti consumatori di sostanze e non, evidenzia:

- che il farmaco maggiormente prescritto nei due gruppi è stato il tramadolo;

- che la diagnosi prevalente per il dolore è, in entrambi i gruppi, la lombalgia;

- che in tutti i casi di prescrizione non compare in cartella la misurazione dell'intensità di dolore;

- che la prescrizione continuativa si è verificata in percentuali alte in entrambi i gruppi;

- che in entrambi i gruppi si evidenzia una quasi assente rivalutazione clinica dell'efficacia dei trattamenti;

- che il gruppo dei consumatori di sostanze ha maggiormente richiesto un incremento del dosaggio degli analgesici oppioidi e una associazione con FANS e adiuvanti;

- che un controllo a campione al fine di valutare l'aderenza alla terapia analgesica oppioide eseguito sul 33\% di consumatori di sostanze in trattamento ha dimostrato che il $100 \%$ degli stessi non assumevano la terapia prescritta.

Più in generale I'analisi dei dati dimostra come i pazienti con anamnesi positiva per abuso di sostanze stupefacenti rappresentino un gruppo a rischio per abuso, misuso e diversione del farmaco analgesico prescritto. Questo pone la necessità di sviluppare delle metodiche operative condivise ed omogenee che coinvolgono più figure professionali per la presa in carico di consumatori di sostanze affetti da sindromi dolorose, al fine di garantire I'appropriatezza prescrittiva e la sicurezza clinica dei pazienti stessi.

Lo studio ha permesso inoltre di tracciare gli elementi di criticità della gestione della terapia oppiacea all'interno del contesto carcerario, specie nella popolazione dipendente da sostanze, suggerendo dei possibili aggiustamenti nel sistema organizzativo in modo da favorire l'appropriatezza e la sicurezza prescrittiva dei farmaci oppiacei per il trattamento del dolore. 


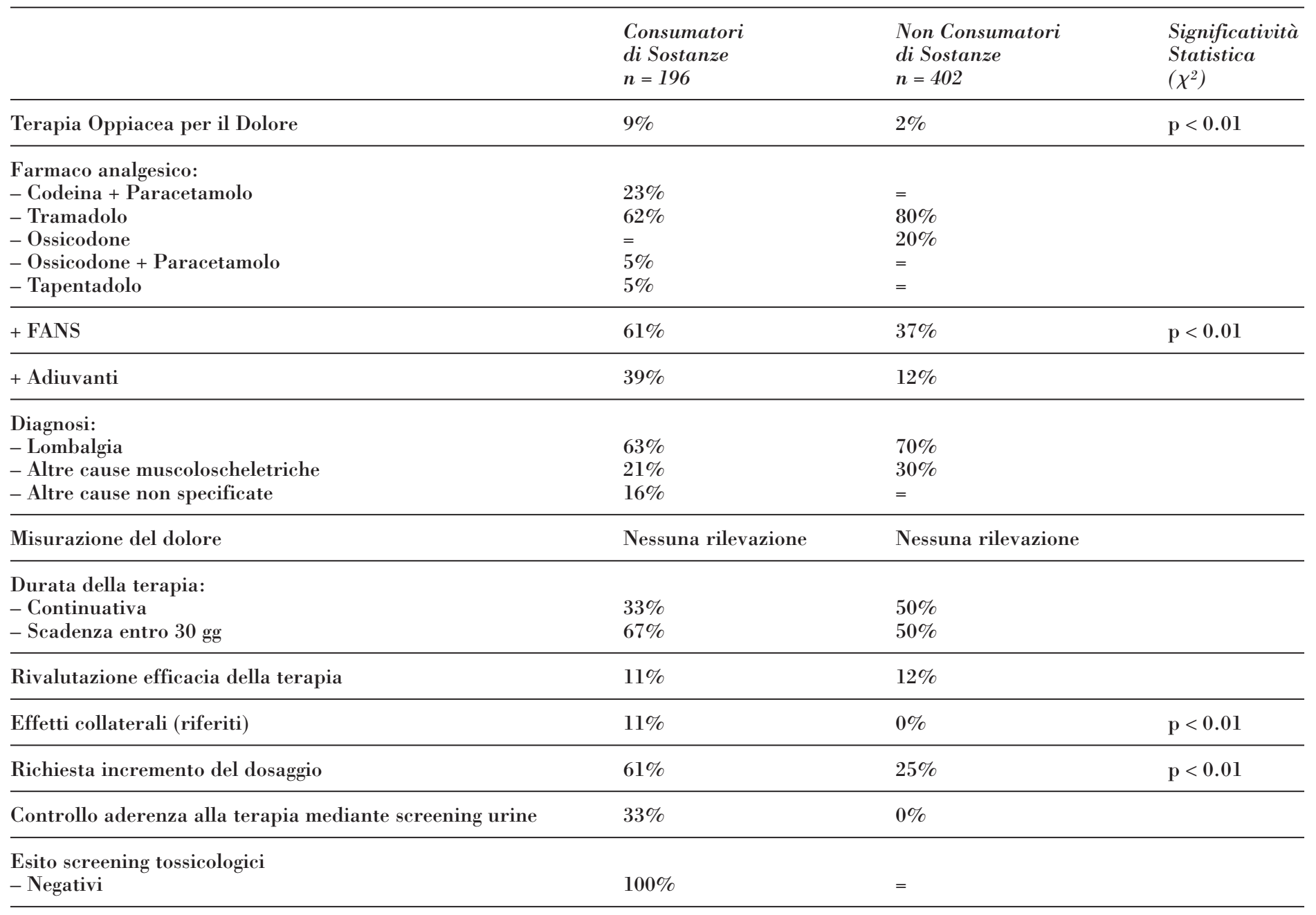

\section{I presupposti e i principi che possono garantire l'e- sigibilità delle cure per il trattamento del dolore in ambito penitenziario}

Uno dei maggiori limiti e ostacoli nell'implementazione della terapia oppiacea per il dolore in ambito penitenziaria è il fenomeno del misuso e della diversione (NHS, 2015b). Essi possono essere superati attraverso un miglioramento dell'appropriatezza prescrittiva e attraverso lo sviluppo di raccomandazioni per l'utilizzo dei farmaci oppiacei per il trattamento del dolore che abbiano lo scopo di:

- fornire indicazioni più precise sulle caratteristiche dei trattamenti farmacologici oppiacei per la terapia del dolore (fra cui ad es. I'introduzione e I'utilizzo di abuse deterrent formulation);

- facilitare la comunicazione e la relazione fra prescrittore e paziente circa i rischi e benefici della terapia con questi farmaci;
- ottimizzare l'efficacia e la sicurezza del trattamento farmacologico attraverso frequenti monitoraggi;

- ridurre i rischi associati alla terapia a lungo termine con gli oppiacei.

Per la stesura di un percorso di presa in carico si devono, infatti, considerare:

- i punti critici decisionali nella gestione di pazienti con dolore cronico candidati alla terapia con oppiacei (identificazione dei fattori di rischio e di vulnerabilità riferiti allo sviluppo di abuso, misuso e diversione);

- il bisogno di costruire una completa e approfondita valutazione del dolore e della diagnosi;

- la necessità di sviluppare procedure flessibili, con un approccio sequenziale dei farmaci, atte a massimizzare l'efficacia (ridurre il dolore, migliorare lo stato funzionale e la qualità della vita) e ridurre le complicanze e gli effetti avversi (come il misuso e la diversione). 


\section{Una proposta di algoritmo in dieci punti per la gestione della terapia con oppioidi per il trattamento del dolore cronico non oncologico in ambito penitenziario}

PUNTO 1
VALUTAZIONE INIZIALE GLOBALE
La valutazione del dolore è un processo multidimensionale e l'approccio al paziente con dolore in carcere deve essere finalizzato alla "presa in carico" globale della persona (attraverso un approccio multiprofessionale e la realizzazione di un programma terapeutico individualizzato)

\section{PUNTO 2 \\ VALUTAZIONE DELL'ADDICTION RISK \\ E DIAGNOSI ALCOLOGICA}

Valutazione dell'addiction risk

Valutazione psicologica del dolore
La diagnosi algologica nel paziente in carcere deve comprendere come primo passaggio la valutazione dell'addiction risk e successivamente la valutazione psicologica e della causa che origina il dolore.

La valutazione algologica è un processo dinamico che presuppone lo sviluppo da parte del terapeuta di competenze di "comunicazione" e di "relazione"

Identificare, nell'intera popolazione detenuta, i soggetti con rischio di dipendenza, diversione, misuso ed overdose

Valutare le seguenti componenti psicologiche del dolore:

- significato e afflizione del dolore

- risposte del paziente per far fronte allo stress e al dolore

- effetti del dolore sulle attività di vita quotidiana

- effetti psicosociali e spirituali del dolore

- effetti psicologici e comportamentali indotte dal dolore (ansia, depressione, ecc.)

- variabili culturali e fattori etnici che possono influenzare la percezione del dolore

- aspettative/credenze del paziente circa i metodi di gestione del dolore

- preferenze ed attitudini del paziente nel ricevere informazioni sul dolore

Rivalutazione nel tempo la diagnosi algologica

Il dolore è una complessa risposta soggettiva e non può prescindere, neanche in ambito penitenziario, dal processo di autovalutazione. Esso può essere reso più genuino dallo sviluppo da parte del terapeuta di competenze di "comunicazione" e "relazione". In ogni caso la misurazione del dolore deve sempre avvenire attraverso I'utilizzo di scale validate, atte anche a valutare gli episodi di riacutizzazione
Valutazione dell'intensità, della tipologia e dell'adattamento al dolore
Il processo di misurazione del dolore deve prevedere come presupposto:

- fiducia del paziente nel curante

- attendibilità e credibilità del prescrittore

- attitudine del curante di entrare in "comunicazione" e "relazione" con il paziente

- accurata indagine anamnestica e approfondito esame obiettivo

- uso di scale validate di misurazione del dolore

- richiesta di esami strumentali

- impostazione di un piano terapeutico antalgico

- precoce e periodica rivalutazione

Definizione del tipo di dolore

- nocicettivo (somatico o viscerale)

- neuropatico

\section{PUNTO 3}

VALUTAZIONE DEL PROGRAMMA DI TRATTAMENTO

Valutazione del programma di trattamento di tipo farmacologico
Per stabilire un programma terapeutico per il trattamento del dolore questo deve essere reso descrivibile, misurabile e trattabile

La terapia farmacologica del dolore prevede un approccio progressivo con la "scala degli analgesici" come suggerito dall'OMS. Essa deve rispettare i principi di:

- informazione e di consenso da parte del paziente

- efficacia nel sopprimere e prevenire il dolore

- scelta della via di somministrazione più efficace e semplice

- scelta di una via alternativa

- prevenzione e trattamento dei possibili effetti collaterali

Il trattamento del dolore non si deve limitare alla sola terapia farmacologica ma considerare, se di utilità, I'aggiunta di eventuali terapie fisiche e comportamentali (anche se nei contesti carcerari essi non possono essere sempre disponibili)
Valutazione del programma di trattamento di tipo fisico e/o comportamentale
Una volta stabilità l'ipotesi trattamentale, all'interno di un progetto terapeutico globale, deve essere stabilito il rapporto rischio/benefico che in ambito penitenziario è declinato soprattutto in termini di rischio dello sviluppo di fenomeni di dipendenza, misuso e diversione 
PUNTO 5

IDENTIFICAZIONE DEGLI OBIETTIVI

TERAPEUTICI
Un programma terapeutico deve essere sempre accompagnato dall'individuazione (condivisa con il paziente) di obiettivi pertinenti, realistici e raggiungibili a breve termine (anche in presenza di condizioni di dolore cronico). È utile sempre evitare l'individuazione di obiettivi a lungo termine, poiché le aspettative del paziente mutano con il tempo e in base alle esperienze e alla risposta ai trattamenti

\section{PUNTO 6}

CONDIVISIONE DELLA TERAPIA

CON IL PAZIENTE (IL CONTRATTO

TERAPEUTICO)
Il progetto terapeutico deve essere concordato con il paziente e deve prevedere il raggiungimento di obiettivi chiari e definiti (il cui raggiungimento può essere misurato). In ogni caso è sempre utile formalizzare con il paziente un contratto terapeutico in grado di responsabilizzarlo e renderlo consapevole dei possibili effetti collaterali ed avversi

\section{PUNTO 7 \\ FASE INIZIALE DEL TRATTAMENTO E AGGIUSTAMENTO DEL DOSAGGIO INIZIALE}

Obiettivi della fase iniziale del trattamento
La fase iniziale del trattamento prevede di cominciare con la somministrazione di basse dosi e valutare l'associazione con FANS ed adiuvanti (anticonvulsivanti, antidepressivi, corticosteroidi, neurolettici e miorilassanti). Il farmaco analgesico va sospeso in caso di assenza di analgesia, di comparsa di effetti collaterali importanti e di mancanza di miglioramento funzionale. Gli oppiacei ad immediato rilascio possono essere utilizzati solo per la titolazione della dose, mentre la "dose di soccorso" va somministrata solo nei casi di breakthrough pain

Fino a 8-12 settimane dall'inizio del trattamento:

- identificare i fattori che scatenano o peggiorano il dolore

- identificare i fattori che alleviano il dolore

- valutare le attività che distraggono il paziente dal dolore

- trattare adeguatamente l'insonnia, I'ansia e la depressione

- prevenire e gli effetti collaterali

- utilizzare i farmaci adiuvanti

\section{PUNTO 8 \\ FASE DI STABILIZZAZIONE \\ DEL TRATTAMENTO}

La durata della prescrizione

La gestione degli effetti collaterali
La prescrizione di farmaci oppiacei non dovrebbe superare i 28 giorni nei soggetti con addiction risk e in tutti i detenuti (consumatori di sostanze o meno) è necessario attuare delle strategie di monitoraggio almeno su base mensile.

Quando la terapia inizia ad essere inefficace, nonostante gli opportuni adeguamenti, o si evidenziano l'insorgenza di effetti collaterali prima non presenti, si consiglia di effettuare la rotazione del farmaco

Le prescrizioni devono avere una durata massima di un mese e devono prevedere una valutazione di efficacia (sull'analgesia) e di sicurezza (sui comportamenti aberranti come il misuso e la diversione e gli effetti collaterali)

Gli effetti collaterali più comuni dei farmaci oppioidi sono stipsi, nausea (vomito) e sedazione e, con frequenza inferiore, confusione mentale, disforia, vertigini, allucinazioni, incubi, prurito, sudorazione, ecc. Essi devono essere sempre farmacologicamente trattati

\section{PUNTO 9}

VALUTAZIONE DELL'ADERENZA ALLE PRESCRIZIONI
L'aderenza alle prescrizioni deve essere particolarmente attenta nella popolazione detenuta e deve consistere nel medical management e nel monitoraggio tossicologico almeno su base mensile

\section{PUNTO 10 \\ CONCLUSIONI FINALI}

Soddisfacenti - continuare:

- dosi stabili

- effetto su analgesia

- no abusi ed effetti collaterali

Fallimento - sospendere se:

- richiesto importante aumento di dosaggio

- no effetto su analgesia

- abusi

- misuso

- diversione

- effetti collaterali

- assenza di compliance 


\section{Conclusioni}

Lo studio condotto presso la Casa di Reclusione di Padova ha messo in evidenza come il trattamento del dolore con farmaci oppiacei deve avvenire con la necessità di un approccio razionale basato sulle evidenze scientifiche e la realizzazione di procedure e raccomandazioni. Quest'ultime devono prevedere una precisa valutazione e monitoraggio della terapia (Adams et al., 2004; Solanki, Koyyalagunta, Shah, Silverman, \& Manchikanti, 2011) e I'utilizzo ove possibile di farmaci con abuse deterrent formulation (Alexander, Mannion, Weingarten, Fanelli, \& Stiles, 2014) e soprattutto I'integrazione dei trattamenti con interventi psicosociali ed educativi (Eccleston et al., 2014).

\section{Bibliografia}

Adams L.L., Gatchel R.J., Robinson R.C., Polatin P., Gajraj N., Deschner M., Noe C. (2004). Development of a self-report screening instrument for assessing potential opioid medication misuse in chronic pain patients. Journal of Pain and Symptom Management, 27(5): 440-59. doi: 10.1016/j.jpainsymman.2003.10.009.

Alexander L., Mannion R.O., Weingarten B., Fanelli R.J., Stiles G.L. (2014). Development and impact of prescription opioid abuse deterrent formulation technologies. Drug and Alcohol Dependence, 138: 1-6. doi: 10.1016/j.drugalcdep.2014.02.006.

Breivik H., Collett B., Ventafridda V., Cohen R., Gallacher D. (2006). Survey of chronic pain in Europe: prevalence, impact on daily life, and treatment. European Journal of Pain (London, England), 10(4): 287-333. doi: 10.1016/j.ejpain.2005.06.009.

Chou R., Fanciullo G.J., Fine P.G., Adler J.A., Ballantyne J.C., Davies P., ... Miaskowski C. (2009). Clinical Guidelines for the Use of Chronic Opioid Therapy in Chronic Noncancer Pain. Journal of Pain, 10(2). doi: 10.1016/j.jpain.2008.10.008.

Croft M., Mayhew R. (2015). Prevalence of chronic non-cancer pain in a UK prison environment. British Journal of Pain, 9(2): 96-108. doi: 10.1177/2049463714540895.

Davis C.S., Carr D. (2016). Physician continuing education to reduce opioid misuse, abuse, and overdose: Many opportunities, few requirements. Drug and Alcohol Dependence, 163: 100-7. doi: 10.1016/j.drugalcdep.2016.04.002.

Department of Health (2007). Clinical Management of Drug Dependence in the Adult Prison Setting Including Psychosocial Treatment as a Core Part.
Eccleston C., Fisher E., Craig L., Duggan G.B., Rosser B.A., Keogh E. (2014). Psychological therapies (Internet-delivered) for the management of chronic pain in adults. The Cochrane Database of Systematic Reviews, (2), CD010152. doi: 10.1002/14651858.CD010152.pub2.

Fazel S., Hope T., O'Donnell I., Jacoby R. (2001). Hidden psychiatric morbidity in elderly prisoners. The British Journal of Psychiatry?: The Journal of Mental Science, 179(6): 535-9. doi: 10.1192/bjp.179.6.535.

Griffin M.L., McDermott K.A., McHugh R.K., Fitzmaurice G.M., Jamison R.N., Weiss R.D. (2016). Longitudinal association between pain severity and subsequent opioid use in prescription opioid dependent patients with chronic pain. Drug and Alcohol Dependence, 163: 216-221. doi: 10.1016/j.drugalcdep.2016.04.023.

Levy M. (2012). Safer prescribing for prisoners. BMJ (Clinical Research Ed.), 344, e447. -- Retrieved from www.ncbi.nlm.nih.gov/pubmed/22282531.

Martel M.O., Finan P.H., McHugh R.K., Issa M., Edwards R.R., Jamison R.N., Wasan A.D. (2016). Day-to-day pain symptoms are only weakly associated with opioid craving among patients with chronic pain prescribed opioid therapy. Drug and Alcohol Dependence, 162: 1306. doi: 10.1016/j.drugalcdep.2016.02.047.

NHS (2015a). Pain Management Formulary for Prisons: Implementation Guide.

NHS (2015b). Pain Management Formulary for Prisons: The Formulary for acute, persistent and neuropathic pain.

Public Health England (2013). Managing persistent pain in secure settings.

Reid K.J., Harker J., Bala M.M., Truyers C., Kellen E., Bekkering G.E., Kleijnen J. (2011). Epidemiology of chronic non-cancer pain in Europe: narrative review of prevalence, pain treatments and pain impact. Current Medical Research and Opinion, 27(2): 449-62. doi: 10.1185/03007995.2010.545813.

Singleton N., Bumpstead R., O'Brien M., Lee A., Meltzer H. (1998). Psychiatric morbidity among adults living in private households, 2000. London: Office for NationalStatistics, 1-164. -- Retrieved from file:///C:/Users/hp/Downloads/psychmorb_tcm77-144477.pdf.

Solanki D.R., Koyyalagunta D., Shah R.V., Silverman S.M., Manchikanti L. (2011). Monitoring opioid adherence in chronic pain patients: assessment of risk of substance misuse. Pain Physician, 14(2): E11931. -- Retrieved from www.ncbi.nlm.nih.gov/pubmed/21412377.

Turk D.C., Swanson K.S., Gatchel R.J. (n.d.). Predicting opioid misuse by chronic pain patients: a systematic review and literature synthesis. The Clinical Journal of Pain, 24(6): 497-508. DOI: 10.1097/ AJP.0b013e31816b1070.

Wasan A.D., Butler S.F., Budman S.H., Fernandez K., Weiss R.D., Greenfield S.F., Jamison R.N. (2009). Does report of craving opioid medication predict aberrant drug behavior among chronic pain patients? The Clinical Journal of Pain, 25(3): 193-8. doi: 10.1097/AJP.0b013e318193a6c4. 